BIO Web of Conferences 1, 00038 (2011)

DOI: $10.1051 /$ bioconf $/ 20110100038$

(C) Owned by the authors, published by EDP Sciences, 2011

\title{
Visual Vection does not Perturb Squatting Posture
}

\author{
Houzaillefata Mohamed ${ }^{1}$ Gilles Dietrich ${ }^{2,1}$ Blandine Bril ${ }^{1}$ \\ (1)Ecole des Hautes Etudes en Sciences Sociales, Groupe de recherche Apprentissage et \\ Contexte, Paris, France \\ (2)Université Paris Descartes, Paris, France \\ houzaillefata.m@gmail.com, blandine.bril@ehess.fr, gilles.dietrich@parisdescartes.fr
}

\begin{abstract}
Vision contributes fundamentally to the control of the standing posture. The illusion of self motion falsely perceived (vection) increases postural sway while standing. In this paper we examine the effect of vection on both standing and deep squatting with the hypothesis that the squatting posture should not be disturbed by the conflict of sensory information due to vection. The results show that standing posture only was affected by the visual stimuli. The widespread use of squatting for work as well as rest could be due in part to this lack of effect of sensory perturbation on postural stability.
\end{abstract}

\section{Introduction}

It is well-known that vision provides an important source of information for the control of posture. When vision is occluded or when visual information contradicts other sources of information, it has been shown that postural sway increases $[1,2,3]$. On the other hand it has been suggested that deep squatting, a near universal everyday posture, squatting, was not perturbed in no vision condition $[4,5]$. These results suggest that vision is not necessary for postural control when squatting. To test this hypothesis, participants were subjected to visual vection. In such a condition the posture is destabilized as the person perceives visually induced self motion even though the body is not actually moving [6] and should not be disturbed when squatting. Vection induces a conflict of sensory information: visual information specifying movement while somatosensory and vestibular information specify a motionless body.

\section{Material and method}

\section{Participants}

Fifteen young adults (mean age: 27 years, range 20 to 41) able to maintain a squatting position easily for a few minutes participate in the experiment. All participants gave informed consent after they have been explained the nature of the experiment.

\section{Material}

A force plateform constituted of two rigid footpads which dimensions were $36 \times 13 \mathrm{~cm}$ was used to record the center of pression (CoP) of each participant The resolution of the foot pad is $1.5 \mathrm{~g}$ and the accuracy ( $\mathrm{x}-\mathrm{y}$ error) of $0.1 \mathrm{~mm}$, sampling rate, $40 \mathrm{~Hz}$.

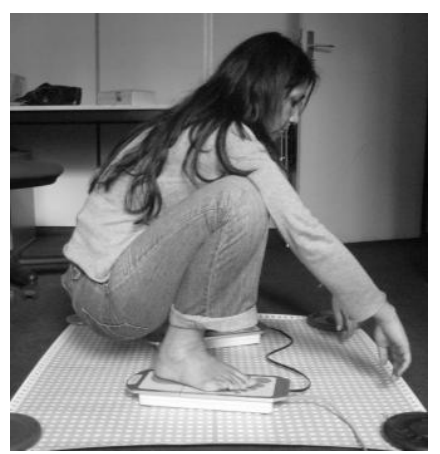

Figure 1. Example of a participant maintaining the deep squatting posture with a free feet position.

\section{Protocol}

Participants stood barefoot, relaxed, arms rested alongside for standing trials and for squatting trials, the only constrain was not to touch the ground with the hands. Each participant performed 20 trials of 35 seconds each. Four visual conditions of a dot pattern composed of 512 white dots on a black screen (figure

2 ) were proposed to the participants. :

- pattern in extension (expansion rate $2 \%$ )

This is an Open Access article distributed under the terms of the Creative Commons Attribution-Noncommercial License 3.0, which permits unrestricted use, distribution, and reproduction in any noncommercial medium, provided the original work is properly cited. 
- pattern in contraction

- pattern in a sinusoidal movement at a frequency of $0.3 \mathrm{~Hz}$

- pattern still.

An additional situation was a situation without vision.

The experiment took place in a small room $(4 \mathrm{~m}$ by 2 meters). The dot pattern was projected on the front wall ( $2 \times 2$ meters). The center of the dot patter was position at eye level for both standing and squatting. The participant was standing at a distance of 3 meters from the front wall.

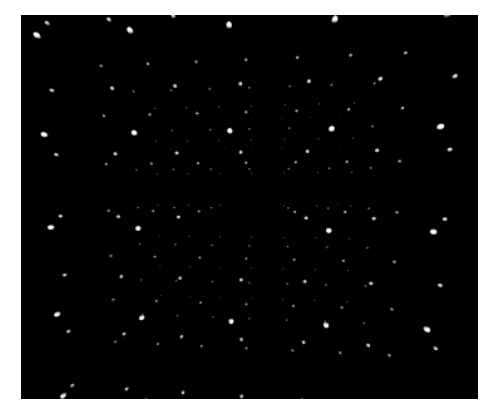

Figure 2. Dot pattern used in the experiment.

For the standing posture the position of the feet was the standard position (feet at $30^{\circ}$ ). For the squatting posture participants could choose a feet position they were comfortable with. The experimental session started with either standing or squatting. Then the five situations were proposed randomly, twice each, for each posture.

\section{Data analysis}

CoP classical and diffusion parameters were computed. Statistical analysis is based on ANOVA, with additional post-hoc (LSD Fisher) tests.

\section{Results}

The data confirm the very significant differences found in previous experiments [4-6]: for all participants the classical postural parameters (length, velocity, surface) of the displacement of the CoP were significantly smaller when squatting compared with standing. Figure 3 gives an example of the displacement of the CoP when squatting that contrast with the displacement of the CoP when standing.

A position by Vection ANOVA shows significant differences between squatting and standing in all classical parameters (length, surface, velocity). In squatting the surface covered by the displacement of the $\mathrm{CoP}$ is in average about 8 times smaller than in the standing position.
In standing the visual stimulation had no significant effect for any of the classical parameters, except for the sinusoidal stimulation which induced a longer $\mathrm{CoP}$ trajectory and a higher velocity but failed to reach significance $(\mathrm{p}=0.062)$.
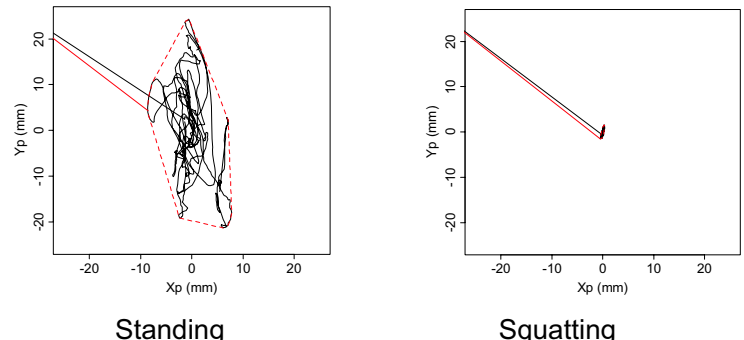

Figure 3. Center of pressure displacement for the sinusoidal stimulation for a typical participant

The dynamic of the sway movement was analyzed owing to the stabilogram diffusion parameters proposed by Collins \& de Lucas [8]. The computation of the stabilogram diffusion function for squatting showed a very small diffusion compared to standing and it was not possible to determine the two phases brought to light in standing condition. That is why we computed the mean square displacement for two given time intervals $\Delta(\mathrm{t})$ at 1 second and 10 seconds along both the anteroposterior and medio-lateral axis.

The results showed a very significant difference

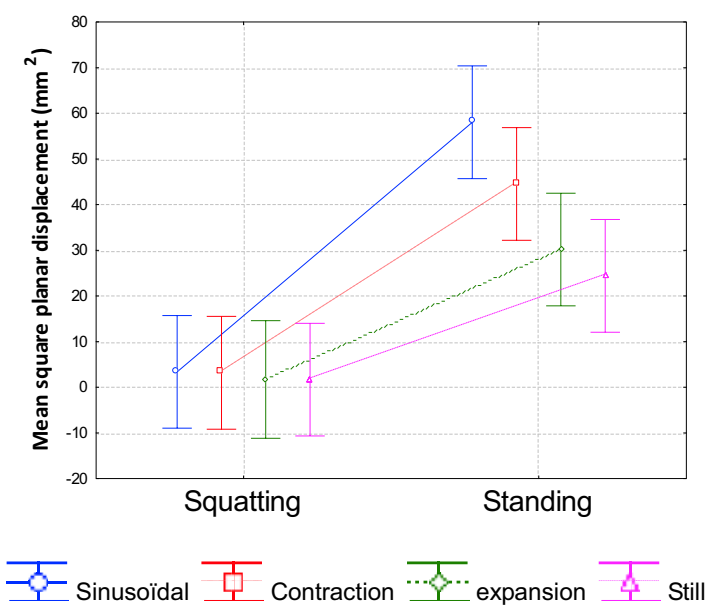

Figure 4. Amplitude of the mean square displacement of the $\operatorname{CoP}(x, y)$ for squatting and standing for the different vection conditions.

The visual stimulations had significant differential effects on the mean square displacement of the $\mathrm{CoP}$ at $1 \mathrm{~s}$ and $10 \mathrm{~s}$ for the standing posture but no effect was 
observed on the squatting posture (see figure 4).

\section{Discussion}

The present study contrasts the effect of a conflict of sensory information due to visual vection in two postures standing and squatting. The absence of any disturbing effect of visual vection on the squatting posture confirms our hypothesis. This result suggests that squatting is either more controlled or more constrained. Compared with standing deep squatting is a posture that considerably decreases the number of degrees of freedom. Hence being more mechanically constrained the need of active postural control is very much reduced, and consequently may necessitate only very little sensory information.

This result emphasizes the importance of broadening investigation about motor action to real world situation. Indeed if squatting is so widely present in many cultural settings, it is most probably due to its adaptive benefit for daily living.

\section{References}

[1] van Asten, W. N., Gieten, C. C., \& Denier van der Gon, J. J. (1988). Postural adjustments induced by simulated motion of differently structured environments. Experimental Brain Research(73), 371-383D. Jones, A.B. Smith, and E.F. Roberts. Book Title, Publisher, 1900 .

[2] Lee, D. N., \& Lishman, J. R. (1977). Vision - The most efficient source of proprioceptive information for balance control. Agressologie, 83-94.
[3] Cornilleau-Pérès, V., Shabana, N., \& Droulez, J. (2005). Measurement of the visual contribution to postural steadiness. Gait \& Posture(22), 96-106

[4] Bril, B., \& Ferrufino, L. (2005). Squatting: a universal resting posture that minimises instability? Gait and Posture, 21 (Suppl. 1), S34

[5] Bril, B. Ferrufino, L. \& Dietrich, G. (2007). Is visual information vital to maintain a squatting posture. Proceedings of the $14^{\text {th }}$ International Conference on Perception \& Action, Yokohama, apan, July 1-6 .

[6] Bril, B., Ferrufino, L. \& Dietrich, G. (submitted) Squatting: a universal posture? The adaptive benefit of squatting for daily living.

[7] Fushiki, H., K. Kobayashi, et al. (2005). Influence of visually induced self-motion on postural stability. Acta Otolaryngol, 125(1), 60-64.

[8] Collins, J.J, \& Collins, J. J., \& De Luca, C. J. (1993). Open-loop and closed-loop control of posture: a random-walk analysis of center-of-pressure trajectories. Exp Brain Res, 95(2), 308-318. 